\title{
Superconducting Effects in Optimization of Magnetic Penetration Thermometers for X-Ray Microcalorimeters
}

\author{
T. R. Stevenson ${ }^{1}$, M. A. Balvin ${ }^{2,3}$, and S. R. Bandler ${ }^{4}$, S. E. Busch, K. L. Denis, W.-T. Hsieh, D. P. Kelly, W. \\ Merrell, P. C. Nagler, J.-P. Porst, J. E. Sadleir, G. M. Seidel, S. J. Smith
}

\begin{abstract}
We have made high-resolution x-ray microcalorimeters using superconducting MoAu bilayers and $\mathrm{Nb}$ meander colls. The temperature sensor is a Magnetic Penetration Thermometer (MPT). Operation is similar to metallic magnetic calorimeters, but instead of the magnetic susceptibility of a paramagnetic alloy, we use the diamagnetic response of the superconducting MoAu to sense temperature changes in an $x$-ray absorber. Flux-temperature responsivity can be large for small sensor heat capacity, with enough dynamic range for applications. We find models of observed flux-temperature curves require several effects to explain flux penetration or expulsion in the microscopic devices. The superconductor is nonlocal, with large coherence length and weak pinning of flux. At lowest temperatures, behavior is dominated by screening currents that vary as a result of the temperature dependence of the magnetic penetration depth, modified by the effect of the nonuniformity of the applied field occurring on a scale comparable to the coherence length. In the temperature regime where responsivity is greatest, spatial variations in the order parameter become important: both local variations as flux enters/leaves the film and an intermediate state is formed, and globally as changing stability of the electrical circuit creates a Meissner transition and flux is expelled/penetrates to minimize free energy.
\end{abstract}

Index Terms-About four key words or phrases in alphabetical order, separated by commas.

\section{INTRODUCTION}

TT HAS long been realized that a sensitive thermometer can be $l_{\text {built based upon the temperature dependence of the }}$ magnetic penetration into a superconductor. Many groups have explored this concept [1-5], but difficulties in obtaining

Manuscript received October 9,2012. This work was supported in part by the NASA ROSES-APRA program and the GSFC IRAD program,

T. R. Stevenson, M. A. Balvin, K. L. Denis, W.-T. Hsieh, and J. E. Sadleir are with NASA Goddard Space Flight Center, Greenbelt, MD 20771 USA (corresponding author phone: 301-286-1221; fax: 301-286-1672; e-mail: thomas.r.stevenson@nasa.gov).

S. B. Bandler is with the University of Maryland, College Park, MD 20742 USA. (e-mail: simon.r.bandler@nasa.gov).

S. E. Busch (e-mail: sarah.e.busch@nasa.gov) and W. Merrell (e-mail: willie.merrell@nasa.gov) are with Oakridge Associated Universities, Oakridge, TN 37830 USA.

D. P. Kelly is with Muniz Engineering Inc., Seabrook, MD 20706 (e-mail: daniel.p.keily@nasa.gov).

P. C. Nagier (e-mail: peter.c.nagler@nasa.gov), J.-P, Porst (e-mail: jp.porst@nasa.gov), and G. E. Seidel (e-mail: George_Seidel@brown.edu) are with Brown University.

S. J. Smith is with the University of Maryland Baltimore County, Baltimore, MD 21250. (e-mail: stephen.j.smith@nasa.gov). desirable properties in thin film superconductors have prevented significant experimental success. Instead, groups interested in this approach have tended towards developing what are known as microwave kinetic inductance detectors (MKIDs), that are similar, but are inherently less sensitive to equilibrium temperature changes (this is due to their operation at temperatures far below the superconducting transition temperature and use of very high frequency read-out). Instead they utilize a non-equilibrium scheme with good sensitivity, and excellent multiplexing potential, but which arguably [6] is not conducive to achieving the very highest levels of spectral resolution.

One of the biggest difficulties in designing a highsensitivity Magnetic Penetration Thermometer (MPT) has been controlling the width of the temperature range of the superconducting transition. We have reported a solution to this long-standing challenge that gives a transition width suitable for producing a detector with usable dynamic range [7-8]. We use a MoAu bilayer, with a Tc of $140 \mathrm{mK}$, as our superconducting temperature sensor, just as is used in many transition-edge-sensor microcalorimeters. By subjecting the superconducting sensor film to the non-uniform magnetic field from the meander, we broaden the superconducting-to-normal transition enough for our device to perform as a broadband $\mathrm{x}$ ray detector. Interestingly this broadening has not been as large as might be expected from the inhomogeneous field, due to the long-range coherence within the superconductor, so that the temperature dependence remains strong enough for use as an $\mathrm{x}$-ray microcalorimeter in which $6 \mathrm{keV}$ x-ray events typically produce temperature rises of $\sim 2 \mathrm{mK}$.

We have described our measurements and analysis of the transition width in our MPT microcalorimeters in a conference presentation [9]. Here we present details of that analysis, along with new results using the electromagnetic response kernel of BCS theory to model the inductance of the devices, in which the applied magnetic field varies on a scale similar to the superconducting coherence length.

\section{MPT TeSt DEVICE AND MEASUREMENT APPARATUS}

The design and operation of our MPT microcalorimeters are similar to Metallic Magnetic Calorimeters (MMCs) [10]. In both MPTs and MMCs we use the temperature dependence of the magnetic susceptibility of a metal film to change the 
inductance of a superconducting niobium meander coil coupled to a SQUID sensor. However, instead of the paramagnetic susceptibility of a $\mathrm{Au}: \mathrm{Er}$ alloy, we use the diamagnetic susceptibility of a superconducting MoAu bilayer to sense temperature change in an overhanging $\mathrm{x}$-ray absorber in electrical contact with the sensor film.

Figure 1 shows one of the MPT devices described here. We used a planar niobium meander with $2.5 \mu \mathrm{m}$ line width, 5.0 $\mu \mathrm{m}$ pitch, and $0.45 \mu \mathrm{m}$ thickness. We covered the meander with a $0.32 \mu \mathrm{m}$ oxide insulation layer, and then a MoAu bilayer film $0.38 \mu \mathrm{m}$ thick. The bilayer had a superconducting transition temperature $T_{\mathrm{c}}=140 \mathrm{mK}$, and a residual resistance ratio $R R R=8.1$, with its normal state resistivity dominated by the high conductivity of its $0.33 \mu \mathrm{m}$ thick Au component. Details of the fabrication methods are similar to what we employ for making MMCs [11].

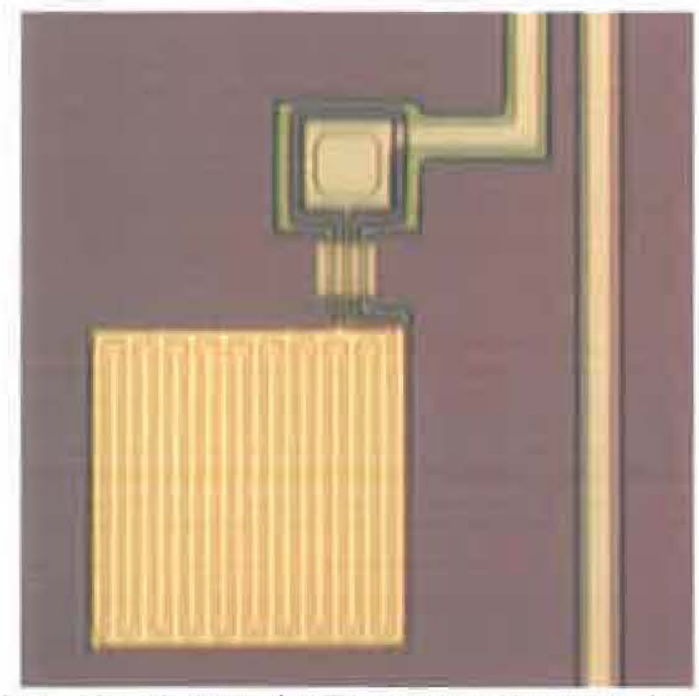

Fig. 1. Magnetic Penetration Thermometer without $\mathrm{x}$-ray absorber. A thinfilm of MoAu bilayer covers a niobium meander coil, with $5 \mu \mathrm{m}$ pitch, in a $100 \mu \mathrm{m} \times 103 \mu \mathrm{m}$ region. An aluminum oxide layer insulates the coil from the bilayer. Niobium microstrip lines from SQUID input and MPT bias circuit (top and right) supply a current to the meander coil with a via through the oxide. The current from the meander returns (at lower left) to the ground of the microstrip in a split path, symmetrically along the top and bottom edges.

We mounted in the MPT devices in a magnetically shielded package in an adiabatic demagnetization refrigerator. With short wire bonds designed to minimize stray inductance, we connected the MPTs to two-stage dc-SQUID amplifiers from PTB-Berlin [12]. At a temperature $>1 \mathrm{~K}$, we stored a persistent current, ranging up to several milliamps, in the niobium circuit biasing a series array of MPTs, then cooled to temperatures as low as $0.030 \mathrm{~K}$. We measured the de level and noise spectra of the flux $\phi$ in the SQUID input at various bias and temperature combinations, and recorded pulses in response to applied x-rays with energies of 1.5 or $5.9 \mathrm{keV}$. Further details of the measurement apparatus are given in [7].

\section{EXPERIMENTAL RESULTS}

During initial cooling after storing a current in the niobium bias circuit, we observed a staircase of flux jumps in the SQUID dc level that was repeatable if the temperature was cycled above $140 \mathrm{mK}$. However, for bias currents $\leq 1.25 \mathrm{~mA}$, the flux-temperature curve was smooth upon warming, and largely non-hysteretic thereafter for temperature excursions below $140 \mathrm{mK}$. At a stored bias current $I_{\mathrm{b}}=1.25 \mathrm{~mA}$, the maximal flux change was $14 \Phi_{0}$. The greatest responsivity to temperature was $\approx 1000 \Phi_{0} / \mathrm{K}$, which occurred near $T=80$ $\mathrm{mK}$. At higher bias currents the peak flux signals decreased. For three different values of the bias current stored in the niobium circuit, the bold curves in Fig. 2 show the SQUID input flux change from high temperature, normalized by bias current, as the MPT was warmed.

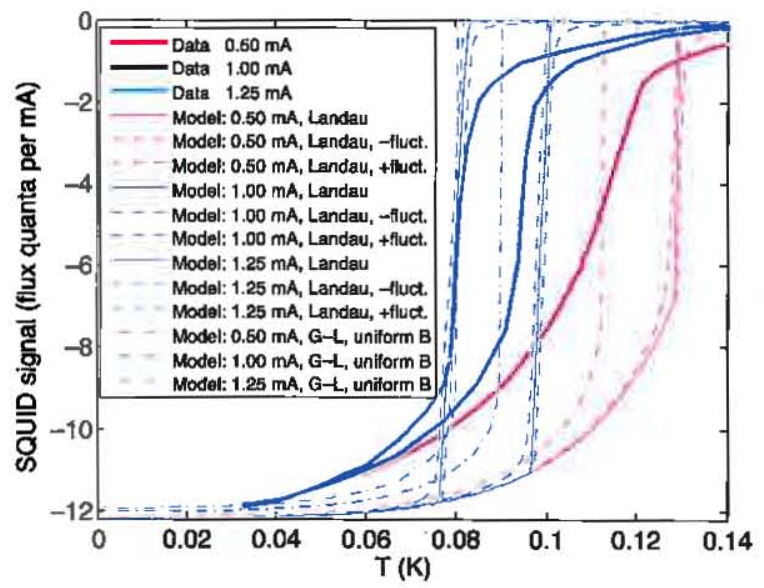

Fig. 2. Measured flux, per unit of bias current, plotted against temperature (bold curves) for stored bias current values $0.50,1.00$, and $1.25 \mathrm{~mA}$. Also shown are various model curves, discussed in Sec. IV.

With the bias circuit wired in a different configuration, we were also able, during a separate refrigerator run, to apply the bias current first at $30 \mathrm{mK}$ and then measure the SQUID flux change during warming to $T>140 \mathrm{mK}$, instead of our normal procedure of cooling after storing the bias current with the $\mathrm{MoAu}$ film in the fully normal state at $\mathrm{T}>1 \mathrm{~K}$. Comparison of the two total flux change values indicated that $\geq 80 \%$ of the flux penetrating the normal bilayer is expelled for $I_{\mathrm{b}}=1.25$ $\mathrm{mA}$ during our normal bias procedure.

Noise measurements [7] with at $I_{\mathrm{b}}=1.25 \mathrm{~mA}$ and $T=80$ $\mathrm{mK}$ showed the presence of no significant excess noise, only SQUID and phonon noise. The phonon noise component extended up to $5 \mathrm{kHz}$, and was consistent with the expected electron-phonon and Kapitza thermal conductance between the bilayer and substrate. The measured ratio of heat capacity to temperature $\approx 1.5 \mathrm{pJ} / \mathrm{K}^{2}$ was similar to the normal state heat capacity of the gold contained in the bilayer, but measurement precision was limited by the accuracy of our measurement of $\mathrm{d} \phi / \mathrm{d} T$ at the dc operating point during the noise measurement.

Noise spectra at $4 \mathrm{~K}$ with the niobium circuit superconducting but the aluminum wire bonds normal gave the total circuit inductance at that temperature to be $4.14 \mathrm{nH}$.

\section{Modeling Methods AND RESUltS}

\section{A. Circuit Model}

From quantization of the flux in the niobium meander coil/SQUID input circuit loop, and the near constant bias 
current supplied to the parallel combination of the SQUID input coil and the MPT meander (enforced by a large ballast inducto: in the bias circuit), one can derive the following relations between the meander coil inductance $L_{\mathrm{m}}(T)$ and the currents $I_{\mathrm{i}}$ and $I_{\mathrm{m}}$ in the SQUID input and meander:

$$
\begin{aligned}
& I_{i}=\frac{-I_{b}\left[L_{m}(\infty)-L_{m}(T)\right]}{L_{m}(T)+L_{i}} \\
& I_{m}=I_{b}-I_{i}=\frac{I_{b}\left[L_{m}(\infty)+L_{i}\right]}{L_{m}(T)+L_{i}}
\end{aligned}
$$

The SQUID input coupling was $\mathrm{Mi}=1 \Phi_{0}$ per $5.4 \mu \mathrm{A}$, and its input inductance $L_{\mathrm{i}}$ was known to be $1.8 \mathrm{nH}$. The inductance of the meander with the MoAu fully normal was computed to be $0.76 \mathrm{nH}$. From $4.14 \mathrm{nH}$ total circuit inductance at $4 \mathrm{~K}$, the value of stray inductance from wirebonds and microstrip was therefore $1.58 \mathrm{nH}$. The total input inductance including strays was $L_{\mathrm{i}}=3.38 \mathrm{nH}$. The overall change in $\phi=-$ $M_{\mathrm{i}} I_{\mathrm{i}}$ between hot and cold limits in Fig. 1 constrains $L_{\mathrm{m}}(0)=$ $0.55 \mathrm{nH}$. Figure 3 shows the experimental meander inductance versus temperature in comparison with models.

\section{B. Screening Currents}

For $I, \leq 1.25 \mathrm{~mA}$, we observe a regime at the lowest temperatures, $\mathrm{T}<60 \mathrm{mK}$, where the MoAu seems to not trap the field applied by the meander, but to expel the flux as completely as possible by means of screening currents. In that regime, the SQUID flux is linear in the stored bias current, i.e. $\phi / I_{b}$ is nearly independent of $I_{b}$.

Measurements of the properties of Transition Edge Sensors made from similar MoAu bilayers have shown that such films exhibit non-local superconducting behavior, with a coherence length $\xi_{0} \approx 0.7 \mu \mathrm{m}$ much larger than their London penetration depth $\lambda_{0} \approx 0.08 \mu \mathrm{m}$ [13]. If we instead treat our MPT bilayer as a local superconductor, we can easily compute the expected meander inductance resulting from screening currents in the bilayer by solving the London equation numerically using the method of Chang [14] (see Fig. 3a). However, we then predict much less variation of the SQUID flux and meander inductance at the low- $T$ end of our data than we observe in Fig. 1 and Fig. $3 b$.

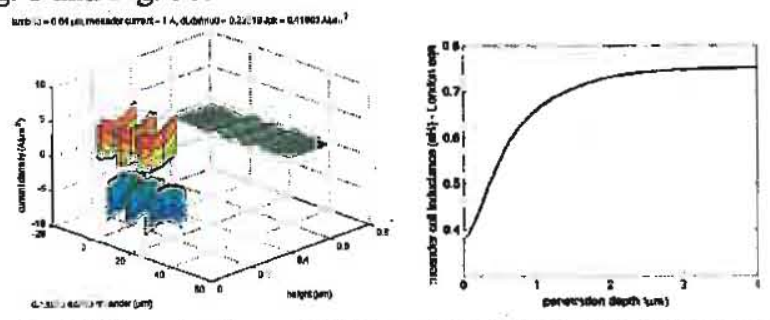

Fig. 3. (a) Example of computed current densities on $\mathrm{Nb}$ and $\mathrm{MoAu}$ film cross-sections for London model. (b) Measured meander inductance versus $T$ and fitted curves for London (local limit) and BCS (non-local) models.

We have computed the expected meander inductance in the non-local limit using the electromagnetic response kernal from BCS theory. We consider a superconducting slab of finite extent in the $\mathrm{z}$ direction and infinite extent in the $\mathrm{x}-\mathrm{y}$ plane.
There is a sheet current pointing in the y direction with spatial variation in the $\mathrm{x}$ direction offset from the superconducting slab in the negative $z$ direction. The current sheet is representative of a meander coil underneath a superconducting body. Our goal is to compute the inductance associated with the current sheet in the presence of the superconducting slab. To do this, we will compute the magnetic field in the region and the supercurrent in the slab and equate the total energy with the energy stored in an inductor.

We start with two equations, Ampere's law neglecting the displacement current and the BCS expression for the supercurrent as the convolution of the BCS integration kernel with the electromagnetic vector potential. The current in Ampere's law is the sum of the sheet current and the supercurrent. Due to the form of the supercurrent, it is mathematically most convenient to work in terms of the vector potential. This is done by replacing the magnetic field in Ampere's law with the curl of the vector potential. We choose to work in the Coulomb gauge so that the curl of the magnetic field becomes the Laplacian acting on the vector potential. The independence of the current on the y direction allows for a simplification of Ampere's law. We can satisfy the gauge condition by allowing only the y component of the gauge field to be non zero and such that it is a function of only the $x$ and $z$ variables. A further simplification can be arranged by expanding the current and vector potential in a Fourier series in $\mathrm{x}$ coordinate. This reduces the differential equation for the vector potential to a one-dimensional equation for each of the z-dependent Fourier modes, with the Laplacian becoming a one-dimensional modified Helmholtz operator. The Helmholtz operator is inverted using its Green's function converting the differential equation into an integral equation. The integral equation is discretized and the solution for the vector potential is found numerically. The solution is used to construct the magnetic field profile and the supercurrent profile, which is used to compute the inductance of the setup as stated above.

The model has three parameters, the superconducting gap of the slab, the Fermi momentum, and quasiparticle effective mass of the superconducting material. The Fermi momentum and quasiparticle effective mass can be expressed in term of the zero temperature London penetration depth and the coherence length, which are more convenient parameters to use. In the absence of independent means for determining the zero temperature London penetration depth and coherence length, the two parameters are fit to the data.

\section{Meissner Effect and Landau Model with Bias Circuit}

At temperatures above $60 \mathrm{mK}$, we observe a bias-currentdependent transition temperature at which the reduction of the meander inductance by effects in the MoAu bilayer rapidly decreases. We interpret this as a manifestation of the Meissner effect specific to the geometry and bias circuit for our devices.

F. London analyzed [15] the Meissner effect for a thin film placed in a uniform applied field in terms of the free energies of the fully normal state and the superconducting state with screening currents (computed in the local limit). Applying 
London's method to our films would predict sudden transitions (above a critical temperature varying with $I_{\mathrm{b}}$ ) to $\phi=$ 0 from a universal low-T curve for $\phi / I_{\mathrm{b}}$. Our observed transitions are softened on both the warm and cold sides.

The transition would be modified if the MoAu energy gap is reduced in response to the magnetic field. As investigated by Douglass [16-17], a simplified Ginzburg-Landau model of this effect predicts that the character of the phase transition changes to second-order if the field increases to the critical value with a sufficiently small ratio of film thickness to effective penetration depth. Curves based on the G-L model used by Douglass, ignoring the spatial variation of field, are compared with our data in Fig. 1. The reduction of the energy gap by the field does soften the flux-temperature curves on the low-T side, but not as much as observed, while on the high- $T$ side the predicted transition is infinitely sharp.

We obtained much better qualitative agreement with the observed transitions using a Landau phase transition model. We assumed that the Type-I MoAu bilayer can enter an intermediate state in which stripes of bilayer go normal (gap drops to zero over a coherence length) as shown in Fig. 4a. When coupled to the bias circuit, we find the free energy difference between the intermediate state and the fully normal state is given by,

$$
\begin{aligned}
& G_{s}-G_{n}-\left(g_{n}(0)-\frac{1}{2} \gamma T^{2}\right)\left(s^{2} d\right)+\frac{\phi^{2}}{2 L_{m}(\infty)}+\frac{\phi^{2}}{2}\left[\frac{1}{L_{m}(\lambda)}-\frac{1}{L_{m}(\infty)}\right](1-f) \\
& -\frac{B_{c}^{2}(T)}{2 \mu_{0}}\left(s^{2} d\right)(1-f)+(\xi-\lambda) \frac{B_{c}^{2}(T)}{2 \mu_{0}}\left(s^{2} d \frac{2 f}{p}\right)
\end{aligned}
$$

where the terms, in order are: the free energy if all the MoAu were fully normal, the magnetic field energy of the meander if the MoAu were fully normal, the extra magnetic and kinetic energy for a fraction (1-f) of superconducting MoAu screening the $\mathrm{Nb}$ coil, the negative of the condensation energy of the superconducting volume of $\mathrm{MoAu}$, and the G-L interface energy between normal and superconducting regions. The length of the sides of the MoAu square are $s$, while $d$ is the MoAu thickness and $B_{\mathrm{c}}(T)$ is the thermodynamic critical field.
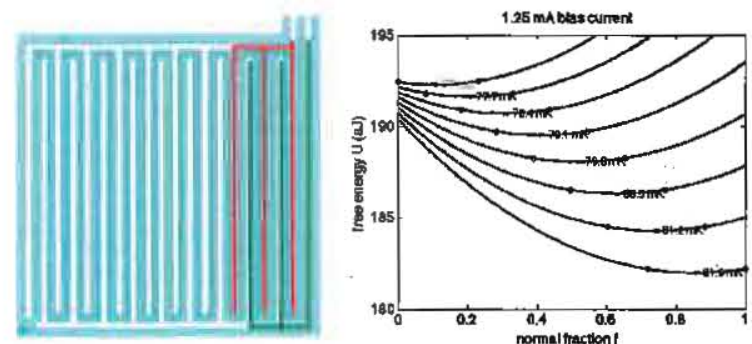

Fig. 4. (a) Postulated intermediate state in MoAu is high-lighted in sketch of $\mathrm{Nb}$ meander coil. (b) Free energy curves at $1.25 \mathrm{~mA}$ bias, for temperatures increasing from 77 to $81.9 \mathrm{mK}$ in $0.7 \mathrm{mK}$ increments, plotted versus fraction $f$ of coil in normal state. Location of minimum free energy is shown by a cross, and range of $f$ values expected from thermal fluctuations is shown by circles.

Our Landau model of the Meissner transition predicts, with reasonable parameters, a finite maximum value for $\mathrm{d} \phi / \mathrm{d} T$ that is of a similar magnitude to the observations at high bias currents (and $T$ substantially less than the zero-field $\mathrm{Tc}=140$ $\mathrm{mK})$. The model requires that the edge barrier for fluxon escape from the MoAu must be small and that pinning of the flux in the bilayer is weak.

\section{CONCLUSION}

MPTs enable x-ray microcalorimeters with zero bias power dissipation and excellent energy resolution. MPTs can be optimized to provide $\mathrm{d} \phi / \mathrm{d} T$ as large as $1000 \Phi_{0} / \mathrm{K}$, with no excess noise, thereby reducing the importance of SQUID noise, provided the sensor film is a Type-I superconducting film with weak flux pinning. The temperature region of steepest $\mathrm{d} \phi / \mathrm{d} T$ represents the Meissner effect in the small device as flux is expelled/penetrates to minimize free energy. The transition width, and microcalorimeter dynamic range, can be increased by non-local superconducting effects when the coherence length is similar to the scale of spatial variations in the field applied by the meander coil.

\section{ACKNOWLEDGMENT}

This work was supported in part by by the NASA ROSESAPRA and the NASA/GSFC IRAD programs.

\section{REFERENCES}

[1] M. V. Moody, H. A. Chan, H. J. Paik and C. Stephens, "A Superconducting Penetration Depth Thermometer", Proceedings of the I7th International Conference on Low Temperature Physics, edited by U. Eckem, A. Schmid, W. Weber, and H. Wuhl (North-Holland, Amsterdam, 1984) pp. 407-408.

[2] D. G. McDonald, "Novel Superconducting Thermometer for Bolometric Applications", Appl. Phys. Lett., vol. 50, 775 (1987).

[3] P. J. Shirron and M. J. DiPirro, "Concept for a high-resolution thermometer utilizing the temperature dependence of the magnetic penetration depth", IEEE Trans. Appl. Supercond. vol. 3, 2140 (1993).

[4] M. J. DiPirro, J. G. Tuttle and P. J. Shirron, "Development of a highresolution penetration depth thermometer", Adv. Cryo. Eng., 1723 (1995).

[5] P. Ranitzsch, "Low Temperature Calorimeters with Superconducting Particle Absorbers", Diploma Thesis, Heidelberg University, HDKIP09-39, 2009.

[6] S. Bandler, E. Figueroa-Feliciano, R. Brissendon, M. Eckart, F. Finkbeiner, K. Irwin, C. Kilbourne, R. Kelley, B. Mazin, F. Scott Porter, D. Schwartz, G. Seidel, S. Smith, and T. Stevenson, "Development of Low-Temperature Detectors For Generation-X and Other Missions Requiring High-Resolution, Large-Format, X-ray Detector Arrays", white paper submitted to Electromagnetic Observations from Space (EOS) Discipline, 2010 astrophysics decadal survey, available from http:/www.cfa,harvard.edu/hea/genx/media/papers/astro2010/Astro2010 Bandler-XMS.pdf

[7] P. C. Nagler, J. S. Adams, M. A. Balvin, S. R. Bandler, K. L. Denis, W.T. Hsieh, D. P. Kelly, J.-P. Porst, J. E. Sadleir, G. M. Seidel, S. J. Smith, T. R. Stevenson, "Performance of Magnetic Penetration Thermometers for X-ray Astronomy," J. Low Temp. Phyśics, 2012.

[8] S.R. Bandler, D. Kelly, P. Nagler, J.-P. Porst, H. Rotzinger, J.E. Sadleir, S.J. Smith, T.R. Stevenson, "Magnetically Coupled Microcalorimeters," J. Low Temperature Physics, 2012.

[9] T.R. Stevenson, M.A. Balvin, S.R. Bandler, K.L. Denis, W.-T. Hsieh, D.P. Kelly, P.C. Nagler, J.-P. Porst, J.E. Sadleir, G.M. Seidel, S.J, Smith, "Magnetic Penetration Effects in Small Superconducting Devices," oral presentation at the 14th International Symposium on Low Temperature Detectors, Heidelberg, Germany, August 1-5, 2011.

[10] A. Fleischmann, C. Enss, and G.M. Seidel, "Metallic magnetic calorimeters", in Cryogenic Particle Detection, 2005, vol. 99 of Topics in Applied Physics, ed. by C. Enss, 151-216.

[11] W.-T. Hsieh, et al., "Fabrication of Metallic Magnetic Calorimeter X-ray Detector Arrays," J. Low Temp. Phys., vol. 151, 357-362 (2008).

[12] D. Drung, C. Assmann, J. Beyer, A. Kirste, M. Peters, F. Ruede, and T. Schurig, IEEE Trans. Appl. Supercond., vol. 17, 699-704 (2007). 
[13] J. E. Sadleir, et al.

[14] W. H. Chang, "Numerical calculation of the inductances of a multisupe:conductor transmission-line system," IEEE Trans. Mag,, vol. 17, 764 (1981).

[15] F. London, Superfluids, Vol. 1, p. 131 (Dover Publications, New York) 1961.

[16] D. H. Douglass, Jr., Phys. Rev. Lett. 6, 346-348 (1961).

[17] R. Meservey and D. H. Douglass, Jr., Phys. Rev. 135, A24-A33 (1964). 